\title{
MARKETING EDUCATIVO COMO ESTRATEGIA DE NEGOCIO EN UNIVERSIDADES PRIVADAS ${ }^{1}$
}

EDUCATIONAL MARKETING AS A BUSINESS STRATEGY IN PRIVATE UNIVERSITIES

\author{
Daniel Sanz Del Vecchio ${ }^{2}$, Tito Crissien Borrero ${ }^{3}$, Jesús García Guiliany ${ }^{4}$ \& Merly Patiño Villanueva ${ }^{5}$ \\ Instituto nacional penitenciario y carcelario" (INPEC)/Universidad de La Costa (CUC)/ Universidad Simón \\ Bolívar. Colombia
}

RECIBIDO: Julio 23 de 2016

ACEPTADO: Noviembre 01 de 2016

\section{RESUMEN}

El presente artículo es resultado de una investigación, cuyo objetivo se direccionó en analizar el marketing educativo como estrategia de negocio, en el sector universitario, específicamente en instituciones privadas en Colombia. Metodológicamente, se aplicó la hermenéutica mediante la revisión documental bibliográfica, y tipo de investigación descriptiva; encontrando que las universidades están dimensionando estrategias de negocio, que facilitan concebir una oportunidad en su mercado, donde es importante la rentabilidad del proceso, además de responder a las expectativas de los clientes reales y potenciales. En este sentido, el sector de las universidades privadas, diseña propuestas particulares accionando su marca institucional, con estrategias de mercado como el CRM, donde consolidan el incremento del valor agregado para el cliente, su sentido de fidelización, y una mejora dentro de la rentabilidad. Concluyendo que el marketing educativo constituye una estrategia de negocio cuando utilizan el CRM, en la medida que se fundamente la capacidad del servicio y la oferta de productos, a favor de un beneficio progresivo y debidamente identificado en el cliente.

Palabras clave: Estrategia de negocio, marketing educativo, CRM, sector universitario.

\begin{abstract}
The present article is the result of an investigation, whose objective was directed to the analysis of educational marketing as a business strategy in the university sector, specifically in private institutions in Colombia. Methodologically, hermeneutics was applied through a bibliographical documentary review, and types of descriptive research; finding that the universities are dimensioning business strategies, which make it easy to conceive an opportunity in their market, where the profitability of the process is important, as well as responding to the expectations of real and potential clients. In this sense, the sector of private universities, designs specific proposals triggering its institutional brand, with market strategies such as CRM, where they consolidate the increase of value added for the customer, their sense of loyalty, and an improvement in profitability. Concluding finally, that educational marketing is a business strategy when CRM is used, to the extent that the capacity of the service and product supply favors a progressive and properly identified customer benefit.
\end{abstract}

Key words: Business strategy, educational marketing, university sector

Este artículo se puede referenciar

Sanz, D. \& Crissien, T., García, J. \& Patiño M. (2017). Marketing educativo como estrategia de negocio en universidades Privadas. En Desarrollo Gerencial Revista de la Facultad de Ciencias Económicas Administrativas y Contables de la Universidad Simón Bolívar-Colombia, 9(1), 160-175.

\footnotetext{
${ }^{1}$ Artículo derivado del proyecto de investigación titulado Gestión Gerencial como acción potenciadora del Marketing Educativo en Universidades Privadas", cofinanciado por la Universidad de la Costa, Barranquilla, Colombia.

${ }^{2}$ Doctor en Ciencias Gerenciales, Docente investigador, Barranquilla, Colombia, correo: d_sanz@hotmail.com.

${ }^{3}$ Doctorante en Educación, Docente investigador Universidad de la Costa, Barranquilla, Colombia, correo: rectoria@cuc.edu.co.

${ }^{4}$ Doctor en Ciencias gerenciales, Profesor de planta Universidad Simón Bolívar, Barranquilla, Colombia, correo: jesus.garcia@unisimonbolivar.edu.co.

${ }^{5}$ Magister en Dirección de marketing, Profesora de planta Universidad Simón Bolívar, Barranquilla, Colombia correo: merlys.patiño@unisimonbolivar.edu.co
} 


\section{1.- INTRODUCCIÓN}

Con el devenir de los tiempos la globalización y el internet han generado transformaciones importantes en las organizaciones y sus procesos, logrando así que las mismas se vean abocadas a realizar adecuaciones tendentes fortalecer sus estrategias para su posicionamiento en el mercado. Por lo que las organizaciones se desarrollan y enfrentan a entornos muy competitivos que cambian continuamente, llegando a definirse incluso como caóticos en algunos casos Prieto, Villasmil y Urdaneta (2011), Prieto y Villa (2011), Prieto, Emoneth, García y González (2015) y Ariza y Pulido (2016). En este sentido, las instituciones de educación superior deben estar en capacidad de afrontar inteligentemente la dinámica generada en los cambios globalizados donde la diversidad en ofertas de estudio es cada vez más amplia, además de ventajas y facilidades las hacen competitivas en el mercado educativo.

En este orden de ideas, Prieto, Burgos, García y Rincón (2016) y Prieto y Villa (2011) expresan que directivos y gerentes han de tomar en cuenta los postulados de mercadeo, el cual es entendido como la actividad que crea, comunica, ofrece e intercambia ofertas que benefician a la organización, a sus grupos de interés (clientes, empleados, proveedores, accionistas, comunidad y gobierno) y a la sociedad en general.

Dentro de este horizonte, las instituciones educativas universitarias desarrollan su actividad en un ambiente sumamente competitivo, complejo, donde se enfatiza en el incremento de la oferta de estudios en la región y fuera de las fronteras de sus territorios nacionales, por lo tanto, la gerencia de las universidades, debe adaptarse a los requerimientos de esta realidad, adecuando el servicio en función a las necesidades de sus demandantes para así cubrir en la mayor medida posible a su satisfacción. Al respecto, García y Pelekais (2012) señalan que son ellos, los altos gerentes, quienes establecen la visión, la misión y la filosofía de la empresa; por tanto, les corresponde dentro del proceso de planificación estratégica, identificar y seleccionar las herramientas a utilizar para posicionarse con ventajas frente a la competencia y contribuir a crear valor.

En este sentido, Manes (2008) plantea como una necesidad que las instituciones de educación incorporen en su estructura al marketing como proceso, que busca oportunidad de negocio. Sobre este particular, es importante acudir al criterio de Zapata (2007), el cual esboza que la mercadotecnia educativa, es un tópico poco abordado para algunas instituciones, sin embargo, lo novedoso en este tema, es precisamente colocarlo al servicio del proceso de crecimiento de las referidas instituciones. Por ello, el interés por el marketing se incrementa de manera significativa en el sector educativo en todos sus niveles.

De allí que, las instituciones educativas sobre todo a nivel universitario, deberían asumir la función del mercadeo orientándola al desempeño de todo el conjunto de actividades destinadas a poner a disposición de los usuarios, los bienes o servicios.. Por su parte, Pinto, Brito y Mendoza (2016), plantean que las 
universidades como instituciones al servicio de la sociedad deben dirigir la mirada hacia un norte estratégico, desarrollando acciones que logren satisfacer las necesidades de su entorno, siendo un factor fundamental para el éxito de sus procesos institucionales

Considerando, estas formas de actuación como el esfuerzo humano sistemático realizado de manera ordenada y de acuerdo con políticas, planes y programas, con el fin de buscar necesidades, definir bienes y servicios, así como seleccionar los mercados de los interesados de fácil acceso, mediante el Customer Relationship Management (C.R.M.) o gestión de relaciones con los clientes.

Al respecto, según Manes (2008), el marketing educativo es el proceso de investigación de necesidades sociales, tendientes a desarrollar y llevar a cabo proyectos educacionales que satisfagan a las mismas, produciendo un crecimiento integral del individuo mediante del desarrollo de servicios académicos ajustados a su valor percibido, disponibles en tiempo y lugar, así como éticamente promocionados para lograr el bienestar de los individuos, además de constituir una oportunidad de negocio para este tipo de organizaciones, utilizando el CRM.

En muchas universidades, sean públicas o privadas, las estrategias de marketing se han vuelto sustanciales para ofrecer un servicio al cliente cada vez más exigente y de acuerdo a las necesidades específicas del interesado, como por ejemplo lo referido por Durán y Parra (2014) al señalar que contar con una estrategia de diversidad brinda a las universidades una mayor habilidad para competir en mercados que también se están volviendo heterogéneos.

Por ello, ofrecer servicios a sus interesados dada su intangibilidad, resulta complejo, porque sencillamente no se puede tocar o palpar; y ello, hace que los servicios ofrecidos a los clientes sean complicados en su estructura, porque intervienen elementos objetivos, pero también subjetivos, difíciles de controlar tales como: la percepción, necesidades, motivación, actitud, lealtad, cariño, fidelidad, los cuales son características complejas de medir a la hora de adquirir un servicio.

Surge entonces como oportunidad de negocio, la implementación del marketing educativo, motivando esto la realización del estudio, con el propósito de analizar el marketing educativo como estrategia de negocio en universidades privadas, ubicándose en el contexto de las universidades colombianas, utilizando la metodología de revisión documental bibliográfica

\section{Mercadeo a nivel Universitario}

Según Carmelo y Puelles (2007), son muchas las universidades que no atienden al tema del mercadeo debido a que la situación es aún muy primaria en términos del marketing que estas aplican. Esto es característico si se parte de un criterio poco innovador, donde las organizaciones educativas privadas no establecen en su norte operativo una estrategia lo suficientemente dinámica para alcanzar un nivel 
adecuado dentro del mercado, cumpliendo con un propósito formativo, pero a su vez respondiendo a la inversión generadora de toda la infraestructura que conforma a la empresa.

En este particular, la UNESCO (2004), en cuanto a la educación superior señala que la evolución de la relación entre el Estado y el mercado en lo que respecta a las responsabilidades de financiación y ordenamiento es un aspecto de repercusión mundial en la educación superior, donde la disminución de los fondos públicos, tienden a asignar un cometido menor al Estado y a los gobiernos en estos asuntos, por lo cual la función y la aportación del sector privado se han ampliado considerablemente, razón por la cual, se está contribuyendo a un fortalecimiento del mercado de la educación superior tanto en los países como mundialmente.

Por lo tanto, esta consideración de la UNESCO (2004), expresa una situación de atención para el mercado universitario privado, más aun cuando las investigaciones realizadas sobre el tema, han demostrado la necesidad que tienen las instituciones universitarias de generar verdaderas prácticas de mercadeo como una estrategia de negocio tangible. Aun cuando, dentro de las áreas de gestión empresarial observada con más recelo por los académicos, en su aplicación a las universidades, ha sido, seguramente, el área de marketing, constituyendo este un contrasentido a la orientación social de la universidad y al flujo libre del conocimiento

Sobre este particular, Pérez (2002) sostiene que existe un escenario acorde para la universidad, donde el enfoque de marketing, contribuiría a su sostenibilidad y desarrollo. En este mismo sentido, Zapata (2007) comenta:

Durante muchos años las instituciones educativas han venido creciendo, gracias a la inercia propia de un mercado poco competitivo. La situación que deben encarar las mismas en la actualidad, tanto en Colombia como en el resto del mundo, es muy diferente. La fuerte e intensa competencia que deben enfrentar, las obliga a responder a una demanda exigente, con propuestas innovadoras, ajustadas a las necesidades del mercado al que se dirigen, (p. 57).

Sobre estas referencias, es posible concretar desde una visión nacional e internacional sobre la educación superior, parece no existir duda, que el tema del mercado, es centro de debate sobre las tendencias actuales, sus perspectivas de desarrollo y las políticas para su conducción. Esto lo corrobora la determinación de Skilbeck (2002), quien plantea que la universidad ya no es más un lugar tranquilo para enseñar, realizar trabajo académico a un ritmo pausado y contemplar el universo, como ocurría en siglos pasados. Es ahora un potente negocio, complejo, demandante y competitivo, que requiere inversiones continuas y de gran escala. 
De ahí que el mercadeo no puede concentrarse únicamente en la divulgación de todos los establecimientos educacionales de la zona, debe, por el contrario, destacar las particulares oportunidades de las instituciones, mediante la ilustración de las potencialidades que se localizan en su organización.

Por ello, es necesario conocer si en las universidades se están cumpliendo acciones de atracción a clientes, si los procedimientos de investigación y exposición a los interesados operan adecuadamente; además de indagar si se procesan los datos producto de los estudios en registros y a su vez, si estos se utilizan para la toma de decisiones y el establecimiento de medidas racionales

En este orden de ideas, The Economist (2010), refiere que:

El modelo de educación superior más orientado hacia el mercado, que ha sido promovido en Estados Unidos y está difundiéndose alrededor del mundo, tiene cuatro grandes ventajas sobre el modelo público. Primero, combina de mejor forma la equidad en el acceso con la excelencia; segundo, se halla en mejores condiciones para producir una mayor diversidad de ofertas educacionales; tercero, es un modelo más sustentable que aquel otro dependiente del sector público, y, cuarto, el hecho de depender de varios patrones otorgaría a las universidades mayor control sobre su propio destino que la dependencia de un solo patrón. (p. 16)

De esta manera es posible observar cómo se ha venido configurando un patrón de trabajo funciona el mercadeo, el cual para el mercado específico de esa región se cuenta sustentado por cuatro elementos que han permitido la solidez esta área de negocio, como lo representa el sector educativo privado, sin embargo, cada una de las regiones a nivel mundial al establecer las particulares necesidades que permitirán orientar a dichos modelos.

De acuerdo a datos recabados por los investigadores, en Colombia, dentro de los esfuerzos identificados en las universidades privadas se evidencian los descuentos en las matrículas a través de acciones de pronto pago, antes de las fechas fijadas para el curso o por grupo de inscritos. Existen zonas del país, donde algunas universidades no sólo ofrecen rebajas en los montos de las matriculas sino también lo complementan con asignaciones, cursos o rifas para promover un interés mayor en los estudiantes.

Huertas (2011), plantea que

La misión de las estrategias del Marketing Educativo es crear y facilitar que los clientes consumidores (estudiantes), puedan interactuar en situaciones de aprendizaje participativo dentro y fuera de contextos educativos, a partir de entender que enseñar supone reconocer la individualidad y la diversidad de los alumnos, en la convicción de que el aprendizaje es un proceso vivencial, fruto de la reflexión, la discusión, el análisis, la interacción y retroalimentación grupal. (p. 8).

Al considerar el concepto de aprendizaje, entonces el Marketing Educativo en las universidades privadas promueve las condiciones para que los estudiantes puedan construir sus conocimientos 
interactuando con medios de comunicación directa y electrónica, caracterizados por la interactividad y por la posibilidad de uso grupal o individual.

\section{Estrategia de Negocio.}

Para Estrada, Saumett \& Osorio (2015) las estrategias se conciben como las acciones estudiadas para alcanzar unos fines, teniendo en cuenta la posición competitiva de la empresa y las hipótesis o escenarios sobre la evolución futura. Asimismo, para Carrión (2007) una estrategia de negocio es aquella que se ocupa de la creación de una posición competitiva y ventajosa para la unidad de negocio dentro de un sector, se comienza con mercado concreto, por lo tanto se relaciona con la toma de decisiones en un negocio o unidad estratégica de negocio y persigue conseguir posiciones competitivas superiores respecto a los competidores, intentando generar capacidades distintivas con el objetivo de conseguir ventaja competitivas sostenibles.

De acuerdo al criterio de los investigadores, la estrategia de negocio permitiría a las organizaciones universitarias privadas, promover acciones para capitalizar los mecanismos los cuales permitan el logro de los objetivos en la institución, teniendo como herramienta fundamental al marketing educativo, esto con base a la necesidad fundamental de este tipo de prácticas, las cuales, de alguna manera, no han sido desarrolladas en la forma requerida.

Por su parte, Kotler (2011), considera que la estrategia de negocio consiste en ser diferente y elegir deliberadamente un conjunto de actividades que permitan crear una combinación única de valor. En definitiva, emprender acciones ofensivas o defensivas para crear una posición defendible en un sector para enfrentarse con éxito a las fuerzas competitivas y de esta manera obtener un rendimiento superior sobre la inversión de la empresa.

Cuando se hace referencia al término marketing educativo como estrategia de negocio, es por el hecho que, si se considera, que las áreas sobre las cuales puede transitar dicha estrategia, se situarán dentro un estilo de diferenciación, en el cual, para los realizadores del artículo, establece una mayor necesidad debido a que los planteamientos estudiados revelan algunas debilidades dentro de este ámbito.

Refiere Kotler (2011), en la práctica, las estrategias de la mayoría de las compañías son una mezcla entre lo planificado acuciosamente, y lo emergente, por ello el proceso de creación de la estrategia no es simple ni está carente de estudios y análisis que deben de llevarse a cabo siempre, si se desea minimizar los riesgos de un fracaso organizacional. La estrategia seleccionada debe aprovechar las fortalezas de la organización y corregir sus debilidades, con el fin de tomar ventaja de las oportunidades externas y, contrarrestar las amenazas del ambiente exterior. 
Las estrategias de negocio, establecen la forma cómo competir en un mercado y negocio en particular, por lo tanto, enfatizan en lo relativo a ventaja competitiva y la asignación de recursos en ella. Por ello, una forma acorde de trabajo para desarrollar una capacidad competitiva, está centrada en el marketing educativo, desde este escenario, será posible conducir una serie de elementos que resultan de interés para lograr cumplir con las metas planteadas en las universidades privadas de Colombia.

Según Manes (2005)

Es necesario entender que hoy la Institución Educativa debe incorporar en su estructura al marketing como proceso y designar un responsable del área al servicio de la comunidad. Sabemos que la comunidad educativa constituye un delicado equilibrio (inestable) de intersubjetividades, por lo tanto, las decisiones de marketing deben ser cuidadosamente evaluadas como alternativas que, bajo un juicio ético, tendrán un impacto social que debe tender a satisfacer necesidades humanas dentro de un proceso de mejoramiento continuo y, por encima de todo, a preservar los valores de la persona, centro de todo accionar y en particular de la educación. (p. 3).

En este particular, el desarrollo de la referida estrategia dentro del sector universitario en Colombia, requiere de un proceso guiado por una visión, misión, para lo cual es necesario establecer planes y programas de acción, además de definir prioridades, recursos y medios disponibles, incluyendo además de una valoración realista del entorno y de las habilidades propias de la institución para lograr esos objetivos o misión. De ahí que esto se logre, no solo con un cuidadoso análisis y valoración, sino con una gran convicción, conocimiento, intuición y profundos valores, por lo tanto, desde la primera idea hasta la última acción del proceso, debe ser asumida con valores de las personas que originan y conciben las estrategias para finalmente ponerlas en ejecútese.

Hablar de valores insertos dentro del mecanismo de una estrategia de marketing, coloca a las instituciones universitarias de Colombia, en un espacio acorde para los usuario y potenciales interesados, logra de esta manera afianzar el respeto que logra desarrollar un proveedor de servicios al corresponder con las especificaciones que fueron ofrecidas en su propuesta, lo cual es un estímulo para lograr la fidelidad de estos.

Es necesario transitar hacia una estrategia de negocio inserta en el modo de mercadeo de las instituciones universitarias en Colombia, más aún cuando se consideran los criterios del Observatorio de la Universidad Colombiana (2011), quienes argumentan que existen docenas de instituciones con vocación de negocio universitario se basan en ofertas variada de carreras que tienen demanda como es el caso de administración e informática, sin que se disponga del registro necesario.

Por lo tanto, es necesario lograr una mayor relación con el cliente del servicio, demostrar que la institución está realmente comprometida con el planteamiento que mercadológicamente se ha colocado, logrando que el mismo no sea creado solo para generar impacto y atractivo orientado a cautivar al 
espectador, por ello debe operarse en un basamento de valores así como de realidades las cuales marquen la firmeza de un trabajo así como de hechos verdaderos para el ofrecimiento logrado.

\section{La estrategia del Customer Relationship Management (Administración basada en la relación con los clientes)}

El término Customer Relationship Management (C.R.M.) o gestión de relaciones con los clientes definido por Greenberg (2002) como un conjunto de procesos de negocio y de políticas de nivel global empresarial que están diseñados para captar, retener y dar servicio a los clientes, lo cual resulta adecuado con el propósito de disponer del marketing como estrategia de negocios dentro de las organizaciones. Por su parte, Thompson (2007) define CRM como el desarrollo y la puesta en práctica de una estrategia empresarial centrada en el cliente significa el dar a sus clientes lo que ellos esperan.

Según Peppers y Rogers (2011), una empresa que se vuelca a sus clientes es una empresa que utiliza la información para obtener una ventaja competitiva y alcanzar el crecimiento y la rentabilidad. En su forma más generalizada, CRM puede ser considerado un conjunto de prácticas diseñadas, simplemente, para poner a una empresa en un contacto mucho más cercano con sus clientes. En el planteamiento de Martínez (2014) se destaca que en la relación proveedor-cliente, éstos desean ir más allá de la simple transacción, dichos clientes buscan una calidad en el servicio que se le ofrece, así como en la relación con su proveedor inmediato hasta el punto que se sienta satisfecho y así se genere valor a la empresa.

Para Qualitas Hispania (2002) en Rosenstiehl (2015) el CRM es una estrategia empresarial que hace del cliente el núcleo central dentro de la organización y su elemento más preciado; que adicionalmente debe gestionar las relaciones con estos a través de varios canales de interacción, coordinando las actuaciones alcanzadas y transmitiendo un mensaje homogéneo, para alcanzar y mantener una relación rentable y de largo alcance.

\section{Desarrollo de una estrategia CRM}

Según el criterio teórico planteado por Schmitt (2004) el proceso CRM contiene cinco pasos, los cuales se muestran a continuación.

Análisis del mundo vivencial de los clientes: el primer paso para el desarrollo de la estrategia CRM es el análisis del mundo vivencial de los clientes. Las compañías deben ser más sensibles a sus clientes e incorporar el conocimiento de estos a sus estrategias e implementaciones. Deben tomar en cuenta la información de los clientes cuando diseñan tanto la experiencia de marca como la interacción con los clientes. 
Figura 1. Los cinco pasos del marco de trabajo CEM

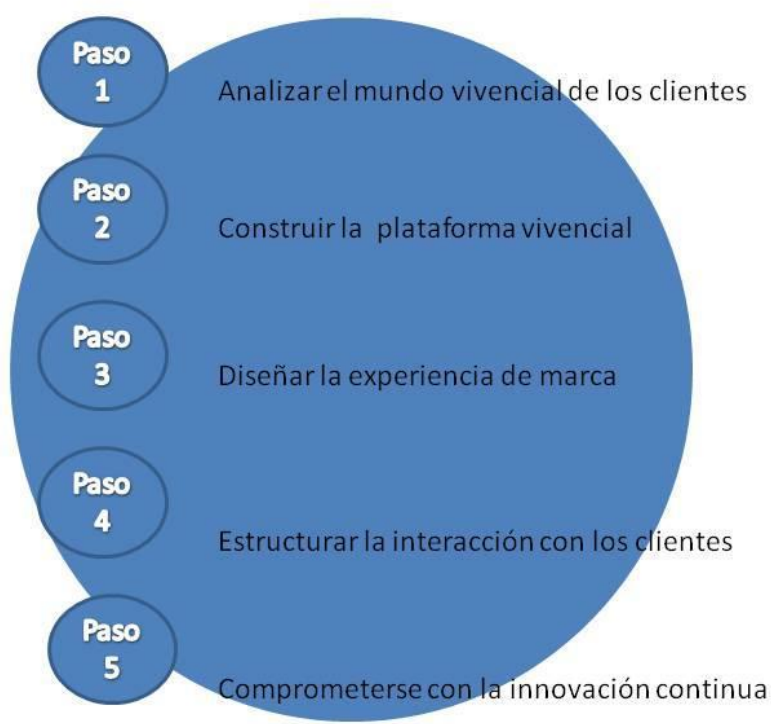

Fuente: Schmitt (2004)

$\checkmark$ Construcción de la plataforma vivencial: El segundo paso del desarrollo de la estrategia CRM es la construcción de la plataforma vivencial; una parte sustancial de la estrategia de negocios y de marketing es lo que los administradores llaman posicionamiento.

$\checkmark$ Diseño de la experiencia de marca: el tercer paso para el desarrollo de la estrategia CRM es el diseño de la experiencia de marca. Schmitt (2004), plantea que la experiencia de marca no es dinámica o personalizada. Planear y diseñar la experiencia de marca exige que se use el tema de la implementación global descrito en la plataforma vivencial. Los clientes encuentran la experiencia de marca en el intercambio directo con el producto o servicio, su apariencia y sensación.

$\checkmark$ Estructuración de la interacción con los clientes: Schmitt plantea que la interacción con los clientes constituye el segundo campo clave en la implementación de la plataforma vivencial y es el cuarto paso para el desarrollo de la estrategia CRM. Así La interacción con los clientes puede mejorar o degradar la experiencia de los mismos clientes a través de la experiencia de marca. Por tanto, es necesario estructurar con cuidado esa interacción, que debe seguir el tema de la implementación global de la plataforma vivencial, y tanto su contenido como su forma deben basarse en las aportaciones de los clientes.

$\checkmark$ Compromiso con la innovación continua: para mantenerse a la altura de lo que promete la plataforma vivencial, la empresa debe realizar innovaciones continuas con el fin de mejorar la experiencia del cliente y mantenerse al ritmo de la competencia. Este es el último paso para desarrollar la estrategia CRM. Para Schmitt la innovación incrementa el valor de hacer negocios de la empresa, mejora la vida de los clientes, pues ofrece soluciones y nuevas experiencias. 
Estos son pasos que deben ser tomados en consideración por toda organización para la consecución de una estrategia, la cual puede tener una mayor dedicación en la especificación de los pasos según sea su necesidad e intención, de esta manera podrá responder con una determinación comprometida con el cliente además del respeto a las condiciones establecidas en la operación o negocio.

\section{2.- MÉTODO}

\section{$\checkmark$ Diseño}

El tipo de investigación, se correspondió a la documental descriptiva, lo que permitió establecer el requerimiento de soporte y veracidad al estudio.

\section{$\checkmark \quad$ Instrumentos}

El estudio estuvo basado en el análisis documental, el cual según Arias (2014) es aquel tipo de investigación cuyo proceso está sustentado en la búsqueda, recuperación, análisis, critica e interpretación de datos secundarios, es decir, los obtenidos y registrados por otros investigadores en fuentes documentales: impresas, audiovisuales o electrónicas.

\section{$\checkmark \quad$ Procedimiento}

El proceso de investigación, implicó la revisión de documentos y fuentes bibliográficas relacionadas con los tópicos de marketing educativo, oportunidades de negocios y CRM, indagando su relevancia dentro de las estrategias utilizadas por las universidades para la prestación de sus servicios

\section{3.- RESULTADOS}

La revisión documental realizada, relacionada con el marketing educativo asumido como una estrategia de negocio, además de los postulados de la herramienta CRM, contextualizado en las universidades de Colombia, plantea una proposición esquemática la cual puede ser generada en estas instituciones como una alternativa viable.

En este sentido, se establece que el C.R.M. es un conjunto de procesos de negocio y de políticas de nivel global empresarial que están diseñados para captar, retener y dar servicio a los clientes, lo cual resulta adecuado con el propósito de disponer del marketing como estrategia de negocios dentro de las organizaciones, siendo para este caso específico las universidades privadas, las cuales de acuerdo a estudios recientes han logrado una presencia en el mercado de alternativas, también han sido objetadas algunas de ellas, como de altos intereses mercantilistas y separadas del compromiso de su oferta.

Sobre este particular, las universidades asumen que la promesa de institución debe ser consistente con la experiencia ofrecida, y por tanto el diseño de la estrategia de marketing debe generarse contemplando los aspectos operativos que la harán realidad. De esta forma, representa un elemento decepcionante 
percibir una realidad diferente detrás de una campaña exitosa, por lo tanto, la experiencia del cliente está en capacidad de afectar a toda la organización razón por la cual debe estar totalmente alineada alrededor del cliente

Otro aspecto de sumo interés para considerar una alternativa en este mercado educativo, es que los clientes del sector demandan sean entendidas sus necesidades reales y se le ofrezcan soluciones para resolver el problema completo, de ahí que las universidades comprometidas en entender y agrupar estas necesidades y entregarlas de manera rápida, lograran la preferencia de los consumidores y los potenciales.

También se destaca, que una estrategia de este tipo no puede estar basada únicamente en intenciones positivas y favorecedoras, para ello, debe existir una plataforma financiera basada en la economía de las relaciones es decir con aquellos indicadores que demuestran el resultado de estas estrategias para no caer en el voluntarismo. Esto indica que la solución de problemas de los clientes deberá reportar una mayor capacidad de respuesta a la consideración de calidad de servicios, lo cual al ubicarse en un nuevo espacio o nivel puede representar un mayor atractivo tanto para los clientes actuales como para los potenciales.

Todas estas intenciones, podrán constituir una realidad para las universidades de Colombia y sus clientes si se dedica el tiempo y recursos a generar experiencias diferentes y diferenciadoras, así como a conocer a la par de medir los indicadores que reflejan el impacto emocional en el cliente. Para esto es vital se generen los mecanismos más acordes, y sea aprovechada en su totalidad la información generada, la cual por lo general se subestima en su contenido.

A pesar de todos los esfuerzos en encuestas, no todas las instituciones aprovechan esta información para generar ventajas competitivas. Es necesario que las encuestas sean diseñadas con intenciones estratégicas y actuar en base a los resultados, además de disponer de la tecnología que permita su conversión de dato a información de valor.

Así mismo, para que el CRM, pueda constituir una opción viable en las universidades, es fundamental poseer un modelo de retribución que pueda de manera transparente reconocer los resultados alcanzados. Por eso la filosofía a seguir establece que, si sólo se retribuye la productividad, nunca se obtendrá la experiencia de cliente, por lo tanto, los empleados deben contar con las herramientas y autoridad necesarias para hacerlo realidad.

Estas consideraciones pueden permitir formular acciones de marketing educativo como estrategia de negocio, asumiendo la estructura del CRM, es decir un proceso conjunto además de alineado el cual repercute de manera positiva en la idealización de los clientes y en la rentabilidad del sector a través de mejoras plenamente identificables. 
Tal como se observa en la figura 2, el conocimiento del cliente es la clave y punto de partida de la estrategia, sería imposible generar iniciativas sin que dichas estrategias sean específicas a sus necesidades y deseos, y aporten valor agregado para demostrar que la universidad ha logrado percibir sus motivaciones. Las decisiones tomadas por las universidades, evidenciadas por sus acciones institucionales, y los resultados que de allí se derivan, actúan como factores contribuyentes para la generación de un mayor conocimiento y mejor calidad percibida del cliente.

Figura 2. Marketing educativo como estrategia de negocio basado en CRM

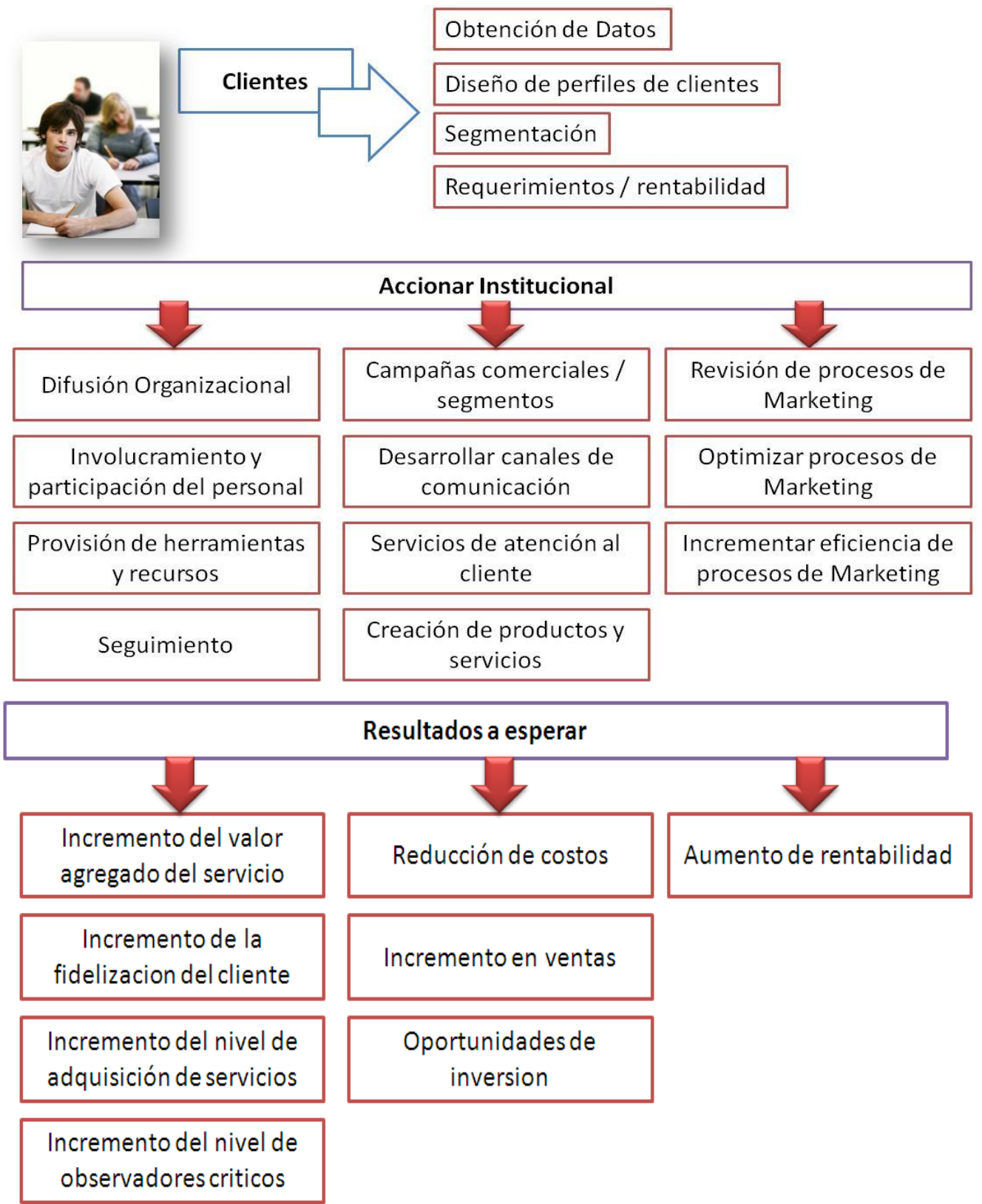

Nota: Elaboración propia, 2016. 
El primer paso para conocer a los clientes es obtener información de los mismos para generar dicho conocimiento por medio de la captura de la información en sistemas eficientes e integrar la misma entre las múltiples herramientas de gestión de la universidad. Integrar estos datos representa un reto tecnológico importante y costoso, estas soluciones ameritan de inversión debido a que las mismas permiten generar datos que provienen de diferentes fuentes, sin necesariamente estar integrados.

Generar perfiles de clientes se orienta específicamente a definir las características demográficas, sus necesidades, deseos, patrones de adquisición y compra, comportamientos de los clientes de forma individual o colectiva. La segmentación de un cliente es un concepto horizontal dentro de la organización que permite que las personas y los sistemas en cada punto de contacto con el cliente, independientemente del canal de comunicación, sepan cómo aportar valor y satisfacer las necesidades del cliente en cualquier momento dado.

Es posible considerar que el desarrollo del marketing educativo dentro de las instituciones universitarias privadas en Colombia, puede constituir una estrategia de negocio viable en beneficio de un cambio que le permita a estas organizaciones ilustrar en una forma más eficiente, las potencialidades de su servicio como proveedor dentro de un mercado sumamente dinámico.

Desde esta perspectiva, a criterio de Durán, Fuenmayor, Cárdenas y Hernández (2016) un mercado competitivo y globalizado no solo se ve reflejado ante las organizaciones destinadas adquirir un interés económico, sino que al igual requiere de la concepción e integración de instituciones académicas, las cuales por ser parte de ese mundo complejo que inunda la actualidad social, requieren utilizar estrategias de marketing, para así garantizar la sustentabilidad y sostenibilidad de ellas en el sector académico

Así entonces, disponer en este caso del marketing educativo, fundamenta el uso de ventajas competitivas existentes dentro de la organización para lograr el propósito de atracción de un colectivo para con el uso de los productos o servicios que ésta promueve, por lo tanto, los resultantes que se planteen obtener a través de la ejecución de este proceso deberán ser cuantificables para el beneficio directo de la institución, más aún dentro de importante sector educativo.

\section{4.- CONCLUSIÓN}

El marketing educativo, debe ser integrado para el caso de las instituciones universitarias privadas de Colombia, como una herramienta para fundamentar su presencia en el mercado nacional e internacional, aun cuando algunas de estas organizaciones, lo han percibido como un elemento de desempeño institucional el cual puede ser manejado desde la perspectiva de la tercerización, lo cual puede no ser el camino más eficiente. 
Así entonces, algunas instituciones universitarias, no han consolidado tales acciones marketing, dejando que el manejo poco responsable de personal contratado, formule ofrecimientos que intentan llamar la atención de los clientes (estudiantes) pero que luego de verificar el ofrecimiento, pueden encontrarse defraudados por el incumplimiento.

De igual manera, se considera que una propuesta fundamentada en el marketing educativo como estrategia de negocio, considerando un basamento en el CRM debe explorar en su proporción de servicio y productos requeridos a los estudiantes, de esta manera será posible operar un cambio progresivo en el bienestar del colectivo así como de la organización basado en acciones sustentable.

\section{5.- REFERENCIAS}

Arias, F. (2004). El proyecto de investigación. Caracas: Editorial Espíteme.

Ariza, O. M. H., \& Pulido, R. A. P. (2016). Cultura organizacional en cooperativas del sector agrícola-en el departamento del atlántico. Libro: Diálogo de saberes desde las Ciencias Económicas, Administrativas y Contables ISBN: 978-958-8557-36-6 ISBN: 978-980-427-011-6. Pp. 6-17.

Benítez, J. P. M. (2014). Relación del proveedor de servicios farmacéuticos con sus clientes: una mirada desde el marketing relacional en el departamento de sucre. En Desarrollo Gerencial Revista de la Facultad de Ciencias Económicas, Administrativas y Contables. Universidad Simón Bolívar, 6(1). P.196-231.

Carrión Maroto, J. (2007). Estrategia: De la visión a la acción, 2da Edición. España: Editorial ESIC.

Durán, S. y Parra, M. (2014). Diversidad Cultural para promover el desarrollo de habilidades sociales en educación superior. Revista Cultura, Educación y Sociedad, 5(1), 55-67.

Durán, S., Fuenmayor, A., Cárdenas, S. \& Hernández, R. (2016). Emprendimiento como proceso de responsabilidad social en instituciones de educación superior en Colombia y Venezuela. En Desarrollo Gerencial Revista de la Facultad de Ciencias Económicas Administrativas y Contables de la Universidad Simón Bolívar-Colombia, 8(2), 58 - 75.

Estrada, H., Saumett, H. \& Osorio, C. (2015). Estrategias de mercado como ventajas competitivas. Libro: Gestión de marketing para el sector agroindustrial. Editorial Universidad Simón Bolívar. ISBN: 978-958-8930-14-5. Pp. 377.

García, J. y Pelekais, C. (2012). La Dirección estratégica como factor potenciador de la Gestión del Conocimiento en Universidades Privadas: una perspectiva desde los Centros de Investigación. Material mimeografiado Jornadas del Centro de Investigación de Ciencias Administrativas y Gerenciales. CICAG. Universidad Dr. Rafael Belloso Chacín. 
Greenberg, P. (2002). CRM at the Speed of Light, Capturing and Keeping. Customers in Internet Real

Time, Segunda Edición. United States of America: Editorial McGraw Hill.

http://www.granadahoy.com/article/granada/619090/quotel/mercado/educativo/es/gran/negocio/mueve/mi llones/eurosquot.html.

Huertas, M. (2010). Marketing Educativo. Recuperado de: http://mshconsultant.net/documentosmsh/articulos/pdf/educativo.pdf

Kotler, P. (2011). Dirección de marketing: Conceptos esenciales. México: Editorial Prentice Hall.

Maind, C. \& Puelles, A. (2007). Marketing universitario. Conceptos y herramientas de marketing aplicadas a la educación superior en España (Tesis doctoral). Universidad Autónoma de Madrid, Madrid, España.

Manes, J. M. (2005). Marketing para Instituciones Educativas. Buenos Aires: Ediciones Granica S.A.

Observatorio de la Universidad Colombiana (2011). Sector de universidades privadas. Análisis 20102011. Recuperado de http://www.universidad.edu.co/

Peppers, D. y Rogers, M. (2011). Managing Customer Relationships: A Strategic Framework. (Second Edition). EEUU: Wiley.

Pérez, B. (2002). Aproximación a un marketing universitario. En Revista cubana de educación superior, $22,(1), 15-36$.

Pinto Aragón, E. E., Brito Carrillo, C. J., \& Mendoza Cataño, C. L. (2016). Equidad económica dimensión de la calidad de vida laboral en la universidad de la Guajira. En Desarrollo Gerencial Revista de la Facultad de Ciencias Económicas, administrativas y contables de la Universidad Simón Bolívar $8(2), 127-143$.

Prieto, Ronald y Villa Carlos (2011). Estrategias de mercadeo interno para optimizar la calidad de servicio en empresas ferreteras. Revista Innovación y Gerencia, Vol. IV, $N^{\circ}$ 2, Octubre, Universidad Dr. José Gregorio Hernández, Maracaibo-Venezuela. Pp. 84-100.

Prieto Pulido, R. A., Villasmil, M., \& Urdaneta, L. (2011). Gestión humana en organizaciones postmodernas. Base fundamental hacia la excelencia organizacional. CICAG, 8(2), 1-12.

Prieto, R., Emonet, P., García, J., \& González, D. (2015). Cambio organizacional como estrategia de gestión en las empresas mixtas del sector petrolero. Revista de Ciencias Sociales, 21(3).

Prieto, R., Burgos, C., García, J., \& Rincón, Y. (2016). Mercadeo interno para optimizar la calidad de servicio en la banca universal. Revista Venezolana de Gerencia, 21(73). 
Rosenstiehl Martínez, J. L. (2014). Herramientas (CRM) en las cooperativas financieras de Colombia y Venezuela para el fortalecimiento de la lealtad de sus clientes. CICAG, 13(1), 161-179.

Skilbeck, M. (2002). The University Challenged: A Review of International Trends and Issues with Particular Relevance to Ireland. Dublin: Higher Education

Smith, L. \& Cavusgil, T. (1984). Marketing Planning for Colleges and Universities. En Long Range Planning. 17, (6), 106.

The Economist (2010). The Brains business: A survey of higher education. September 10: 3-22

UNESCO. (2004). Educación Superior en una sociedad mundializada. Documento de orientación; París: Organización de las Naciones Unidas para la Educación, la Ciencia y la Cultura, p. 10. Recuperado de: http://unesdoc.unesco.org/images/0013/001362/136247s.pdf

Zapata, E. (2007). Mercadeo Educativo. Estrategias para promover instituciones y programas, 2 ed. Bogotá: La librería de la U. 